\title{
ARTICLE
}

Multiple myeloma gammopathies

\section{RUNX proteins desensitize multiple myeloma to lenalidomide via protecting IKZFs from degradation}

\author{
Nan Zhou ${ }^{1}$ Alvaro Gutierrez-Uzquiza ${ }^{1} \cdot$ Xiang Yu Zheng ${ }^{1} \cdot$ Renxu Chang ${ }^{1} \cdot$ Dan T. Vogl $\mathbb{1}^{2} \cdot$ Alfred L. Garfall ${ }^{2}$ \\ Luca Bernabei $\mathbb{D}^{2}$ - Anita Saraf ${ }^{3}$. Laurence Florens ${ }^{3} \cdot$ Michael P. Washburn $\mathbb{1}^{3,4}$. Anuradha Illendula ${ }^{5}$. \\ John H. Bushweller ${ }^{5}$. Luca Busino $\mathbb{D}^{1}$
}

Received: 23 August 2018 / Revised: 14 December 2018 / Accepted: 21 January 2019 / Published online: 13 February 2019

(c) The Author(s) 2019. This article is published with open access

\begin{abstract}
Ikaros family zinc finger protein 1 and 3 (IKZF1 and IKZF3) are transcription factors that promote multiple myeloma (MM) proliferation. The immunomodulatory imide drug (IMiD) lenalidomide promotes myeloma cell death via Cereblon (CRBN)dependent ubiquitylation and proteasome-dependent degradation of IKZF1 and IKZF3. Although IMiDs have been used as first-line drugs for MM, the overall survival of refractory MM patients remains poor and demands the identification of novel agents to potentiate the therapeutic effect of IMiDs. Using an unbiased screen based on mass spectrometry, we identified the Runt-related transcription factor 1 and 3 (RUNX1 and RUNX3) as interactors of IKZF1 and IKZF3. Interaction with RUNX1 and RUNX3 inhibits CRBN-dependent binding, ubiquitylation, and degradation of IKZF1 and IKZF3 upon lenalidomide treatment. Inhibition of RUNXs, via genetic ablation or a small molecule (AI-10-104), results in sensitization of myeloma cell lines and primary tumors to lenalidomide. Thus, RUNX inhibition represents a valuable therapeutic opportunity to potentiate IMiDs therapy for the treatment of multiple myeloma.
\end{abstract}

\section{Introduction}

The major therapeutic goal for multiple myeloma (MM) treatment is complete remission and prolonged survival [1,2]. Small molecules such as the proteasome inhibitor bortezomib and the immunomodulatory imide drugs (IMiDs) thalidomide

Supplementary information The online version of this article (https:// doi.org/10.1038/s41375-019-0403-2) contains supplementary material, which is available to authorized users.

Luca Busino

businol@upenn.edu

1 Department of Cancer Biology, Perelman School of Medicine, University of Pennsylvania, Philadelphia, PA, USA

2 Division of Hematology Oncology, Perelman School of Medicine, University of Pennsylvania, Philadelphia, PA, USA

3 The Stowers Institute for Medical Research, Kansas City, MO, USA

4 Department of Pathology and Laboratory Medicine, The University of Kansas Medical Center, Kansas City, KS, USA

5 Department of Molecular Physiology and Biological Physics, University of Virginia, Charlottesville, VA, USA and lenalidomide, in addition to autologous hematopoietic stem-cell transplantation, have markedly improved overall survival. However, patients with disease refractory to both IMiDs and bortezomib have a median event-free-survival and overall survival of only 5 and 9 months, respectively $[1,2]$. Thus, the search for novel agents to potentiate IMiDs represents an important therapeutic goal.

Ikaros family zinc finger protein 1 and 3 (IKZF1 and IKZF3) are transcription factors of the IKZF family, including also IKZF2, 4, and 5 proteins [3], that function as homodimers and hetero-dimers to regulate lymphopoiesis [4, 5]. Their expression is initiated during the early stages of lymphoid progenitors and increases as lymphocytes differentiate into mature cells [4]. Genetic data has revealed that the loss of IKZF1 and IKZF3 results in a block of lymphoid lineage differentiation and a susceptibility to develop acute lymphoblastic leukemia (ALL) [6]. Consistently, IKZF1 and IKZF3 are frequently mutated tumor-suppressor genes in ALL [7]. In contrast, MM cells display dependency on IKZF1 and IKZF3 for cell-autonomous proliferation [8, 9]. Indeed, the loss of IKZFs by shRNAs or by expression of a dominant-negative IKZF3 mutant inhibits myeloma growth [8, 9].

The IMiDs thalidomide, lenalidomide, and pomalidomide bind to a specific pocket in the E3 ligase Cereblon 
(CRBN) [10, 11], promoting CRBN interaction with the IKZF1 and IKZF3 proteins and favoring their ubiquitylation and proteasome-dependent degradation [8, 9]. IMiDinduced degradation of IKZFs promotes the expression of the interferon gene program essential to repress MYC and proliferation of MM cells [8, 9, 12, 13]. Despite the clinical success of the IMiDs, refractory disease still presents a therapeutic challenge.

In addition to the IKZF family, another family of transcription factors implicated in human hematological cancers is the Runt-related transcription factor family (henceforth referred to as RUNXs). RUNX1 alterations, including translocations, mutations, and gene amplifications, are frequently observed in human leukemia [14-16]. The RUNX proteins (RUNX1, RUNX2, and RUNX3) are transcription factors that bind to promoters and enhancers via the Runt homology domain (RHD), a domain also required for interaction with its partner CBF $\beta$ [17]. RUNX1 targets multiple genes that are pivotal regulators of hematopoiesis, including the hematopoietic-specific member of the E-twenty-six (ETS) family [18]. Although RUNX1 is essential for murine embryonic development and fetal liver hematopoiesis, conditional deletion of Runxl revealed a non-essential role in adult hematopoiesis [19].

Here, we show that RUNX1 and RUNX3 physically interact with IKZF1 and IKZF3 in vitro and in vivo. When complexed with RUNXs, IKZFs become refractory to CRBN-dependent ubiquitylation and degradation induced by IMiDs. Importantly, genetic loss or chemical inhibition of RUNX proteins result in enhanced sensitivity of MM cells to IMiDs. Our data open the possibility of utilizing RUNX inhibition to potentiate IMiD therapy in MM.

\section{Results}

\section{IKZF1 and IKZF3 physically associate with RUNX1 and RUNX3 in MM}

IKZF1 and IKZF3 are transcription factors highly expressed in myeloma that contribute to myeloma cell survival $[8,9]$. To better understand the function of IKZFs, we sought to identify novel physiologic binding partners. To this end, we generated a human MM cell line, ARP-1, stably expressing physiologic levels of FLAG-tagged human IKZF1 or IKZF3 via retroviral delivery. FLAG-peptide eluates from anti-FLAG affinity purifications, either from nuclear extract (nucleoplasm) or benzonase-extracted detergent-insoluble fraction (DNA-bound), were trypsinized and subjected to mass spectrometry analysis for protein identification (Fig. 1a and Supplementary Table 1). Relative to FLAGimmunoprecipitates from ARP-1 cells infected with an empty virus, the peptides corresponding to the NuRD and
SWI/SNF complexes, known members of the IKZF1 and IKZF3 complexes, were identified (Fig. 1b, c) [20, 21]. Surprisingly, we identified the transcription factors RUNX1, RUNX3, and CBF $\beta$ as interactors of both IKZF1 and IKZF3 (Fig. 1b, c).

To validate our proteomic screen, we performed co-immunoprecipitation experiments in both ARP-1 and OPM-1, two MM cell lines, and confirmed the interaction between stably expressed FLAG-IKZF1 and IKZF3 and endogenous RUNX1 and RUNX3 (Fig. 1d). Reciprocal co-immunoprecipitation of endogenous IKZF3 was also observed in FLAG-RUNX1 and RUNX3 immunoprecipitates (Fig. 1e). Antibodies against the endogenous RUNX1/ 3 and IKZF1/3 were validated by siRNAs (Supplementary Fig. 1a). To rule out the possibility of DNA-mediated interaction, we incubated the anti-FLAG-IKZF1 and IKZF3 immunoprecipitates with benzonase to hydrolyze any residual DNA contamination. After extensive washes, we found that RUNX1 was still associated with the IKZFs, suggesting that DNA did not mediate this interaction (Fig. 1f). In agreement with the latter point, purified recombinant IKZF1 and IKZF3 displayed efficient interaction with in vitrotranslated RUNX1 (Fig. 1g).

Since RUNX1 binds to the core binding factor $\beta$ subunit $(\mathrm{CBF} \beta)$ [22, 23], we determined whether IKZFs-RUNX1 form a ternary complex with $\mathrm{CBF} \beta$ (Supplementary Fig. 1b). The interaction between IKZF1 and CBF $\beta$ was detected when RUNX1 was co-expressed, suggesting that the three proteins could form a complex. Importantly, the association of CBF $\beta$ with IKZF1 was not dependent on the ability of IKZF1 to form homodimers. Indeed, expression of FLAG-IKZF1 (1-400), a dimerization-impaired mutant that contains the first four zinc fingers ( $\mathrm{ZnFs}$ ) only [24], retained its ability to interact with CBF $\beta$ in a RUNX1-dependent manner (Supplementary Fig. 1c).

Collectively, these results suggest that IKZFs assemble a novel complex via direct interaction with RUNXs in MM cells. Moreover, IKZF1 utilizes the DNA-binding ZnFs to assemble a ternary complex with RUNX1-CBF $\beta$.

\section{IKZF1 and IKZF3 utilize the N-terminal ZnF domain to associate with the activation and inhibition domain in RUNX1}

The IKZF proteins contain four N-terminal C2H2-type ZnFs involved in sequence-specific DNA binding [20, 24-26], and two additional C-terminal $\mathrm{ZnFs}$ that promote homodimerization and hetero-dimerization with the IKZF family members [24, 27]. To determine the domain required for interaction with RUNX1, we generated a series of N-terminal and C-terminal deletion mutants in IKZF3 (Fig. 2a, b). While deletion of the $\mathrm{C}$-terminal $\mathrm{ZnFs}$ involved in dimerization did not result in loss of RUNX1 interaction (mutants $\Delta 420-509$ 

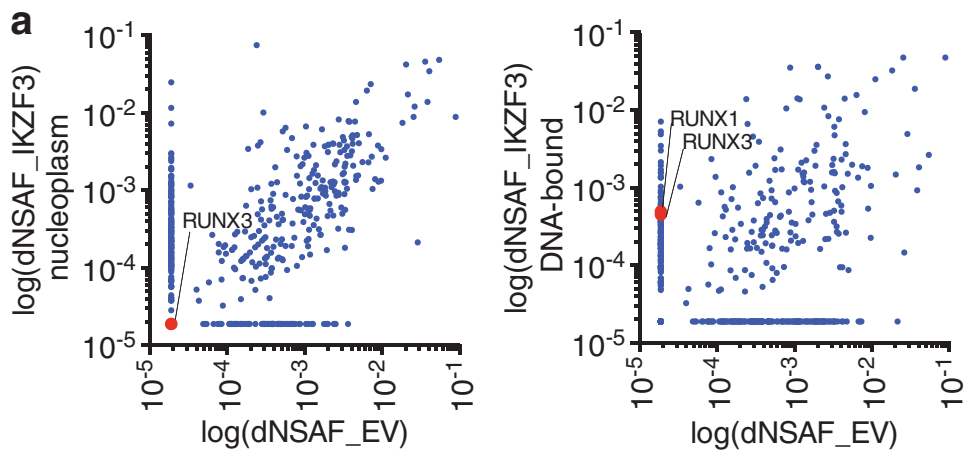

b

\begin{tabular}{|c|c|c|c|c|c|}
\hline Complex & Protein & $\begin{array}{c}\text { IKZF3 } \\
\text { Nucleop. } \\
\text { \#Peptide } \\
\end{array}$ & $\begin{array}{l}\text { IKZF3 } \\
\text { DNA-bound } \\
\text { \#Peptide }\end{array}$ & $\begin{array}{c}\text { IKZF1 } \\
\text { Nucleop. } \\
\text { \#Peptide }\end{array}$ & EV \\
\hline \multirow{9}{*}{ NuRD } & MTA1 & 1 & 2 & 3 & 0 \\
\hline & HDAC1 & 4 & 3 & 6 & 0 \\
\hline & HDAC2 & 3 & 6 & 6 & 1 \\
\hline & RBBP4 & 6 & 2 & 6 & 1 \\
\hline & RBBP7 & 3 & 2 & 4 & 1 \\
\hline & MBD2 & 3 & 2 & 5 & 1 \\
\hline & MBD3 & 0 & 1 & 2 & 0 \\
\hline & CHD3 & 4 & 4 & 16 & 0 \\
\hline & CHD4 & 19 & 24 & 43 & 1 \\
\hline SWI/SNF & SMARCD2 & 0 & 2 & 1 & 0 \\
\hline \multirow[t]{3}{*}{ RUNX-CBF $\beta$} & RUNX1 & 0 & 4 & 0 & 0 \\
\hline & RUNX3 & 1 & 3 & 2 & 0 \\
\hline & $\mathrm{CBF} \beta$ & 1 & 2 & 0 & 0 \\
\hline
\end{tabular}

d
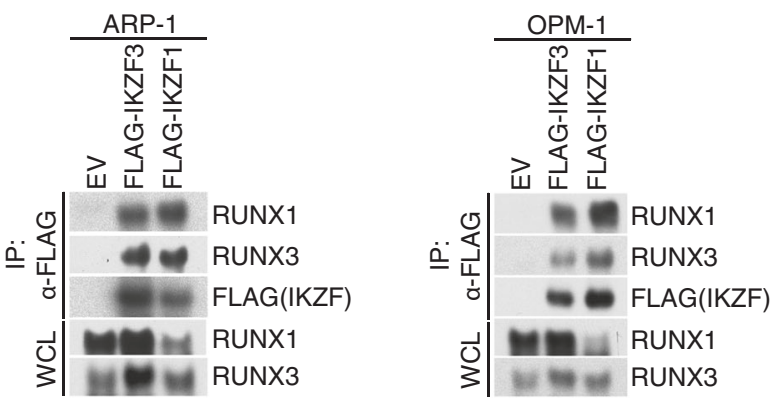

$\mathbf{f}$

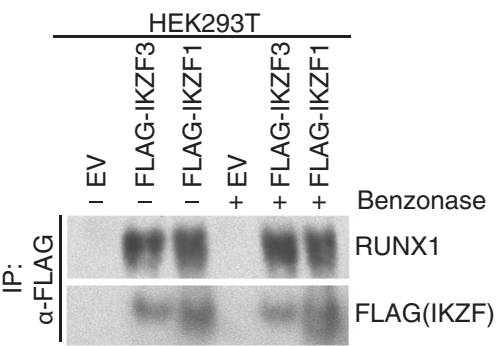

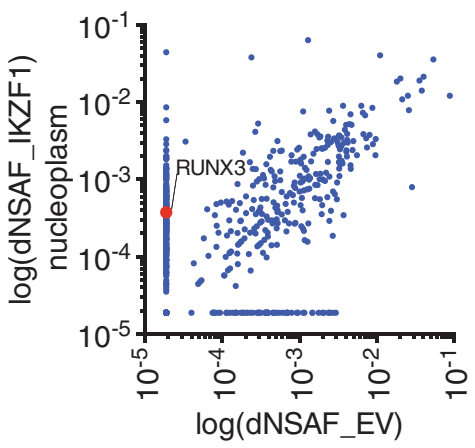

C

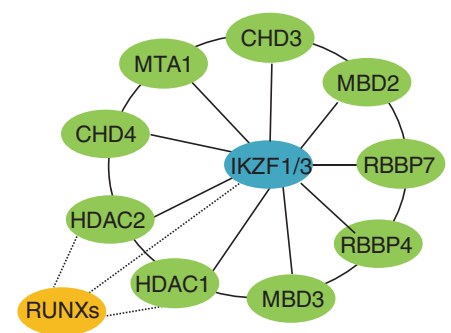

e

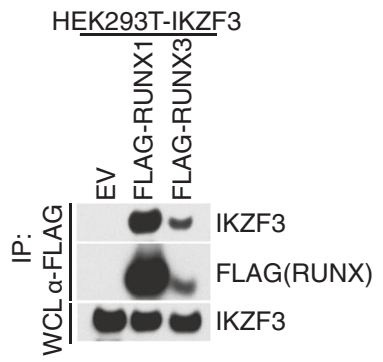

g

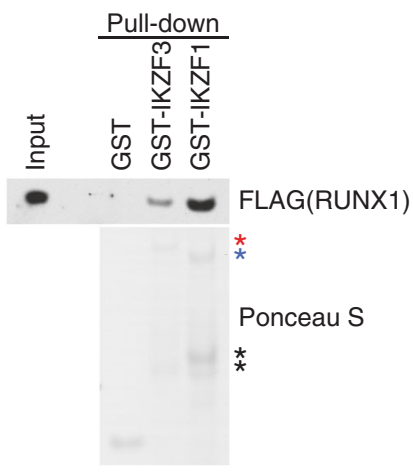

and $\Delta 480-509$ ) (Fig. 2a, b), deletion of the N-terminal ZnFs eliminated IKZF3-RUNX1 association (mutants $\Delta 1-181$, $\Delta$ 1-243 and $\Delta 1-279$ ) (Fig. 2a, b).

In an effort to understand the relevance of individual $\mathrm{ZnFs}$, we assessed the interaction of IKZF3 with mutations in the zinc-coordinating cysteines of each $\mathrm{ZnF}$ to RUNX1
(Fig. 2c). With this analysis, we demonstrated that $\mathrm{ZnF}$ (2) is critical in mediating interaction with RUNX1. To further confirm that IKZF3 N-terminal $\mathrm{ZnFs}$ are directly involved in RUNX1 interaction, we purified IKZF3 containing ZnF (1-4) or ZnF (5-6) and tested the association with RUNX1 in vitro. In agreement with our in vivo mapping (Fig. 2a), 
Fig. 1 IKZF1 and IKZF3 physically associate with RUNX1 and RUNX3 in multiple myeloma. a Scatter plot of distributed normalized spectral abundance factor (dNSAF) in FLAG-immunoprecipitates (IP) from ARP-1 cells stably expressing FLAG-tagged IKZF1 and IKZF3 upon mass spectrometry analysis. For proteins with $\mathrm{NSAF}=0$, the lowest NSAF value was arbitrarily assigned. b List of peptides for the indicated proteins. EV empty vector. c Schematic model of IKZF1/3 interactors. For a complete list of interacting proteins see Supplementary Table 1. d The cell extracts of ARP-1 (left) and OPM-1 (right) cells stably expressing FLAG-tagged IKZF1 and IKZF3 were immunoprecipitated with an anti-FLAG resin and the immunocomplexes were probed with antibodies to the indicated proteins. Specificity of RUNX1 and RUNX3 antibodies was assessed using siRNAs against RUNX1 or RUNX3 (Supplementary Figure 1a). e HEK293T cells stably expressing IKZF3 were transfected with FLAG-RUNX1 or RUNX3. FLAG-immunoprecipitates were probed with antibodies to the indicated proteins. f HEK293T cells were transfected with FLAGtagged IKZF1 or IKZF3. The cell extracts were subjected to antiFLAG IP and the immunocomplexes were treated with Benzonase for $30 \mathrm{~min}$ where indicated. IPs were probed with antibodies to the indicated proteins. g Purified GST-tagged proteins as indicated were incubated with in vitro translated FLAG-tagged RUNX1. GST pulldowns were probed with anti-FLAG antibodies. Ponceau S staining shows the expressions of GST-proteins. The red asterisk indicates GST-IKZF1, blue asterisk shows GST-IKZF3, and black asterisks show cleavage products. Unless otherwise noted, immunoblots are representative of three independent experiments

IKZF3-ZnF (1-4) are sufficient to directly interact with RUNX1, in contrast to IKZF3-ZnF (5-6), which are dispensable (Fig. 2d). Notably, the ZnF (1-4) in IKZF3 are highly similar to the corresponding ZnFs in IKZF1, with $\mathrm{ZnF}$ (2) displaying $100 \%$ sequence homology (Supplementary Fig. 1d). Thus, we interpret that binding results would be identical amongst IKZF1 and IKZF3. Attempts to recapitulate binding in vitro utilizing recombinant single ZnFs resulted in minimal interaction with RUNX1 (data not shown), potentially suggesting that the interaction surface with RUNX1 may have multiple contact points beyond $\mathrm{ZnF}$ (2).

RUNX1 contains an N-terminal conserved DNA-binding domain (called the Runt domain). This region is responsible for both sequence-specific DNA-binding and heterodimerization with $\mathrm{CBF} \beta[22,23]$. At the C-terminus, RUNX1 possesses an activation domain (AD) that interacts with transcriptional co-activators, such as p300/CBP [28] and an inhibitory domain (ID) that counteracts the AD [29]. In an attempt to determine the IKZF3-interacting domain of RUNX1, we performed in vitro Glutathione S-Transferase (GST)-pull down utilizing the IKZF3 ZnF (1-4) fragment fused to GST as bait against in vitro-translated RUNX1 (either full-length or C-terminal truncations) (Fig. 2e, f). Deletion of a $\mathrm{C}$-terminal region containing the $\mathrm{AD}$ and ID resulted in ablation of interaction with IKZF3, suggesting that $\mathrm{AD}$ and/or ID are necessary to promote interaction with IKZF3. To assess sufficiency, we purified recombinant AD and ID (either individually or in combination) fused to GST
(Fig. 2g). Both GST-AD and GST-ID were sufficient to interact with IKZF3 in vitro, although when the AD and ID were combined, the interaction with IKZF3 was greatly enhanced (Fig. 2g). To confirm the requirement for the AD and ID in cells, we performed co-immunoprecipitation experiments using lysates of HEK293T cells expressing constructs encoding FLAG-tagged RUNX1 (Fig. 2h). To minimize non-specific interactions mediated by DNA, we expressed RUNX1 (K83D), a mutant which is incapable of binding DNA [30]. In agreement with our in vitro mapping, simultaneous ablation of $\mathrm{AD}$ and ID resulted in the loss of IKZF3 interaction (Fig. 2e, f), while individual deletion of the AD or ID did not impair, or only partially impaired, the interaction with IKZF3, respectively. Both in vitro and in vivo mapping data suggest that the interaction surface on RUNX1 requires the cooperation of the AD and ID.

Together, our data suggest that IKZF1 and IKZF3 utilize the DNA-binding $\mathrm{N}$-terminal $\mathrm{ZnFs}$ but not the C-terminal ZnFs involved in homo-dimerization and hetero-dimerization, to interact with the AD and ID of RUNX1 (Fig. 2i).

\section{RUNXs inhibit CRBN-dependent IKZF1 and IKZF3 degradation}

Earlier work suggested that the therapeutic effects of the IMiDs reflect a CRBN gain-of-function favoring degradation of IKZFs [8, 9]. The region of IKZF1 that mediates lenalidomide-dependent binding to $\mathrm{CRBN}$ is located within the $\mathrm{ZnF}$ (2) [8, 9], thus overlapping with the identified RUNX1-binding site. These findings led us to hypothesize that RUNXs compete with CRBN for binding to IKZFs.

To test this hypothesis, we first analyzed the levels of IKZF1 in OPM-1 cells depleted of the RUNX proteins. In OPM-1 RUNX1 $1^{+/+} R U N X 3^{+/+}$cells, lenalidomide-induced degradation of IKZF1 but not RUNX1 and RUNX3 (Fig. 3a). While the kinetics was unchanged in the single $R U N X 1^{-1-}$ or $R U N X 3^{-1-}$ knock-out cells, the double knock-out cells displayed an overall accelerated degradation of IKZF1 (Fig. 3a). Importantly, IKZF1 and IKZF3 were the only CRBN-substrates that displayed enhanced degradation in the $R U N X 1^{-/-} R U N X 3^{-/-}$cells upon drug treatment; ZFP91, another CRBN-target [31], showed a slight time-dependent decrease when cells were treated with lenalidomide and no change in the $R U N X 1^{-1-} R U N X 3^{-/-}$ cells (Fig. 3b). Dose escalation experiments and $\mathrm{EC}_{50} / \mathrm{EC}_{80}$ calculation confirmed the accelerated degradation of IKZF1 and IKZF3 in the $R U N X 1^{-/-} R U N X 3^{-l-}$ cells (Fig. 3c and Supplementary Fig. 2a).

To gain mechanistic insights into how RUNX proteins protect IKZF1 from IMiD-dependent degradation, we set out to test whether RUNX1 competes out CRBN for binding to IKZF1. In HEK293T cells treated with 
a

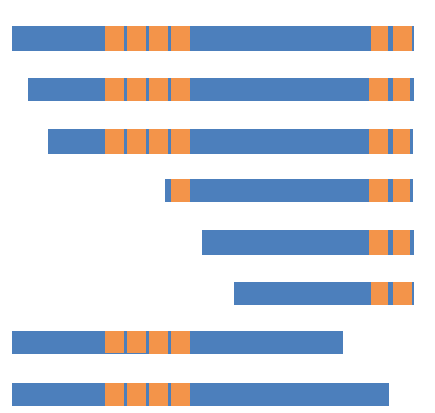

C

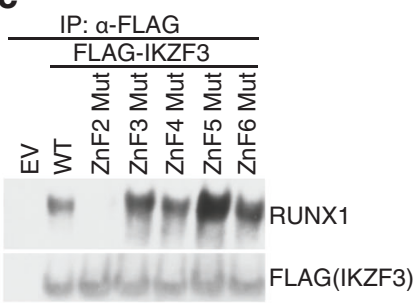

b

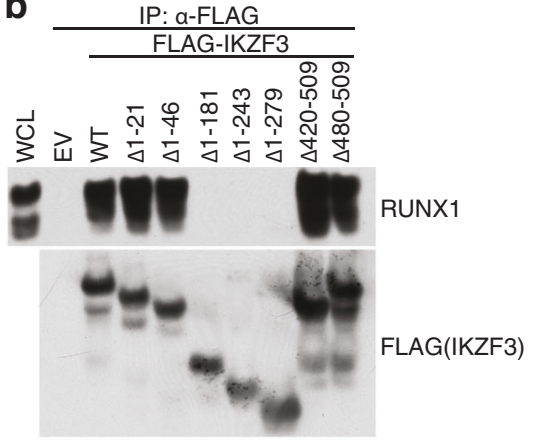

d

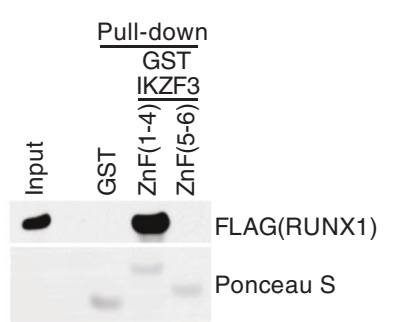

e

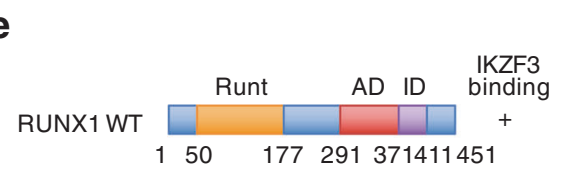

RUNX11-410 $\square+\square+$

RUNX1 1-290

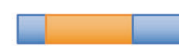

RUNX1 1-250

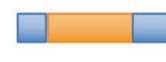

RUNX1 1-210

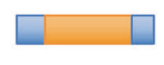

g
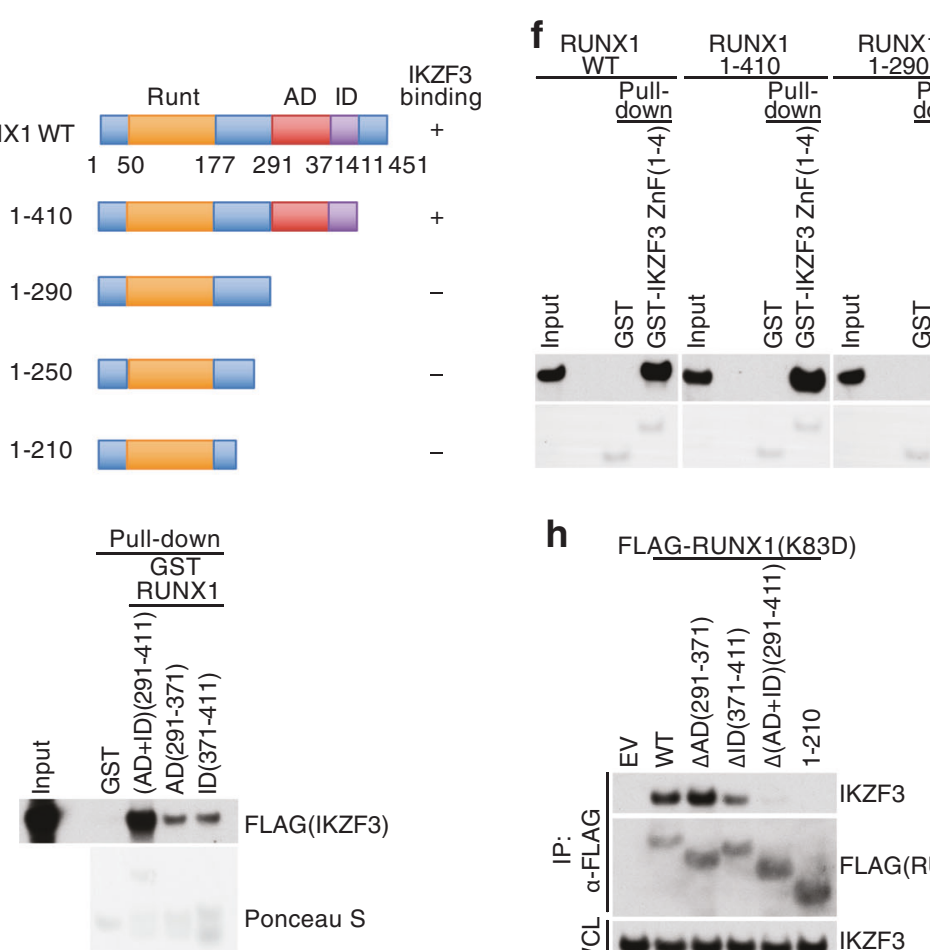

h

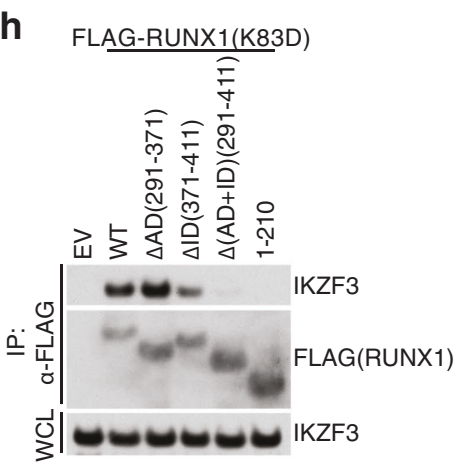

i

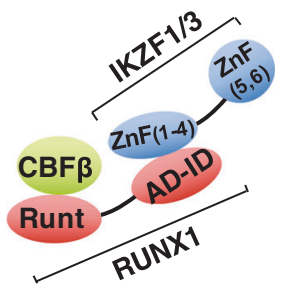

lenalidomide, IKZF1 was effectively enriched in FLAGCRBN immunoprecipitates (Fig. 3d). Importantly, overexpression of RUNX1 abrogated IKZF1 binding to CRBN, in line with the hypothesis that RUNX1 and CRBN compete for the same binding site in IKZF1. RUNX1 not only displaced IKZF1-CRBN interaction, but also blocked CRBN-dependent IKZF1 ubiquitylation (Fig. 3e).

Previous evidences revealed that lenalidomide downregulates IRF4 transcription, connecting this mechanism to the anti-myeloma activity [32-35]. Thus, we assessed whether the dimer RUNX1-IKZF1 is changed by lenalidomide at the IRF4 locus by chromatin immunoprecipitation (ChIP) (Fig. 3f). We confirmed decreased binding of IKZF1 at the IRF4 locus by ChIP in OPM-1 cells treated with lenalidomide. In $R U N X 1^{-/-} R U N X 3^{-/-}$cells, although slightly increased in DMSO-treated cells, IKZF1 downregulation by lenalidomide was more profound, confirming the function of RUNXs in protecting IKZF1 from 
Fig. 2 IKZF1 and IKZF3 utilize the N-terminal ZnF domain to associate with the activation and inhibition domain of RUNX1. a HEK293T cells were transfected with constructs encoding an empty vector (EV), FLAG-tagged IKZF3 wild-type (WT), or mutants. A schematic representation of IKZF3 mutants is shown. IKZF3 mutants that interact $(+)$ or do not interact $(-)$ with RUNX1 are shown. b Immunoblot analysis of FLAG-IKZF3 immunoprecipitation (IP). Immunocomplexes were probed with antibodies to the indicated proteins. c HEK293T cells were transfected with constructs encoding an empty vector (EV), FLAG-tagged IKZF3 wild-type (WT) or mutants as indicated. The cell extracts were subjected to anti-FLAG IP and the immunocomplexes were probed with antibodies to the indicated proteins. d Purified GST-tagged proteins as indicated were incubated with in vitro translated FLAG-tagged RUNX1. GST pull-downs were probed with anti-FLAG antibodies. Ponceau $S$ staining shows the expressions of GST-proteins. e Schematic representation of RUNX1 mutants. RUNX1 mutants that interact $(+)$ or do not interact $(-)$ with IKZF3 are shown. f Immunoblot analysis of GST pull-downs. Purified GST-tagged proteins as indicated were incubated with in vitro translated FLAG-tagged RUNX1. GST pull-downs were probed with antiFLAG antibodies. Ponceau S staining shows the expressions of GSTproteins. $\mathbf{g}$ Same as in $\mathbf{f}$, except that the indicated GST-proteins were used. h HEK293T cells stably expressing IKZF3 were transfected with constructs encoding an empty vector (EV), FLAG-tagged RUNX1 wild-type (WT), or mutants as indicated. The whole cell lysates (WCL) were subjected to anti-FLAG IP and the immunocomplexes were probed with antibodies to the indicated proteins. i Schematic model of the interaction between IKZF1/3 and the AD and ID of RUNX1. Unless otherwise noted, immunoblots are representative of three independent experiments

degradation. Notably, IRF4 and $M Y C$ expression was lower in $R U N X 1^{-/-} R U N X 3^{-/-}$cells upon lenalidomide treatment (Fig. 3g). These data suggest that RUNXs and IKZFs work together at similar genomic sites and that ablation of RUNX proteins results into an amplification of the lenalidomide effect towards IKZF1/3 inhibition.

Next, we assessed the sensitivity of MM cell lines to lenalidomide. First, we assessed protein levels of RUNXs in four lenalidomide-insensitive MM cell lines (ARP-1, OPM-1, RPMI-8226, and KMS-11) and three lenalidomide-sensitive lines (NCI-H299, U266, and MM1S) (Supplementary Fig. 2b) [8]. RUNX1 was overall elevated in the lenalidomide-insensitive MM cell lines and downregulated in the sensitive lines, with KMS-11 being the only exception. Notably, loss of RUNX1 and RUNX3 in OPM-1 (Fig. 3h), ARP-1, and RPMI-8226 (Supplementary Fig. 2c) did not affect cell proliferation as compared to $R U N X 1^{+/+} R U N X 3^{+/+}$ cells. Instead, a significant decrease in proliferation was detectable in the OPM-1 RUNX1 $1^{-/-} R U N X 3^{-/-}$cells upon treatment with lenalidomide (Fig. 3h). These cells displayed sensitivity to $0.1 \mu \mathrm{M}$ lenalidomide, a concentration at which a minimal effect on MM proliferation was detectable (Supplementary Fig. 2d). Similar sensitization was observed in OPM-1 RUNX1 $1^{-/-} R U N X 3^{-/-}$cells upon pomalidomide treatment (Supplementary Fig. 2e) but not with the proteasome inhibitor bortezomib (Supplementary Fig. 2f).
Altogether, these data suggest that RUNX proteins protect IKZFs from CRBN-dependent degradation and genetic ablation of RUNXs sensitizes cells to low doses of IMiDs.

\section{RUNX inhibition dissociates the IKZFs-RUNXs complex and potentiates the cytotoxic effect of lenalidomide in myeloma}

To assess whether chemical inhibition of RUNXs results in changes in RUNXs-IKZFs interaction, we tested the panRUNX inhibitor, AI-10-104 [36]. AI-10-104 interferes with the association of RUNX with $\mathrm{CBF} \beta$, thereby leaving RUNX in an auto-inhibited state. AI-10-104 induced a dose-dependent dissociation of $\mathrm{CBF} \beta$ and IKZF3 from RUNX1 (Fig. 4a) and RUNX3 (Fig. 4b). Importantly, treatment of cells with increasing concentrations of AI-10-104 resulted in increasing RUNX1 and RUNX3 dissociation from IKZF3 (Fig. 4a, b). Similar data were produced when IKZF1 was pulled down (Fig. 4c, d). AI-10-104 did not displace RUNX1-IKZF1/3 interaction at the AD-ID interface (Supplementary Fig. 3a), hence it is possible that AI-10-104 dissociates the RUNX1-CBF $\beta$ complex, resulting in a conformational change ultimately affecting IKZFs interaction.

Next, we tested whether the inhibition of RUNXs could enhance the cytotoxic effect of lenalidomide. To this end, we treated five MM cell lines with combination doses of lenalidomide and AI-10-104. Low doses of lenalidomide had a modest effect on MM cell proliferation (Fig. 4e), however, combination of the two drugs induced an overall proliferation defect in the MM cell lines tested (Fig. 4e). Combination index $(\mathrm{CI})$ calculation revealed that the MM cells expressing low RUNX1 (NCI-H929, MM1S, and U266, Supplementary Fig. 2b) were particularly sensitive to the drug combination, revealing a synergic effect at submicromolar dosage (Fig. 4f). CI calculation for the lenalidomide-insensitive cells lines, OPM-1 and RPMI8226 cells, also displayed synergy, although a higher concentration of both drugs was required. In line with this, combination of AI-10-104 and lenalidomide downregulated the myeloma oncogenes MYC and IRF4 (Supplementary Fig. 3b).

Notably, when used as single agent, AI-10-104 displayed a moderate effect on cell proliferation (Fig. 4e), in line with the genetic evidence that ablation of RUNX1 and RUNX3 had no effect on proliferation of MM cells (Fig. 3h and Supplementary Fig. 2c).

Finally, overexpression of RUNX1 in NCI-H929 cells displaying low levels of endogenous RUNX1 resulted in reduced degradation of IKZF1/3 (Supplementary Fig. 4a, b), as well as cell desensitization to lenalidomide treatment (Supplementary Fig. 4c). Moreover, RUNX inhibition 


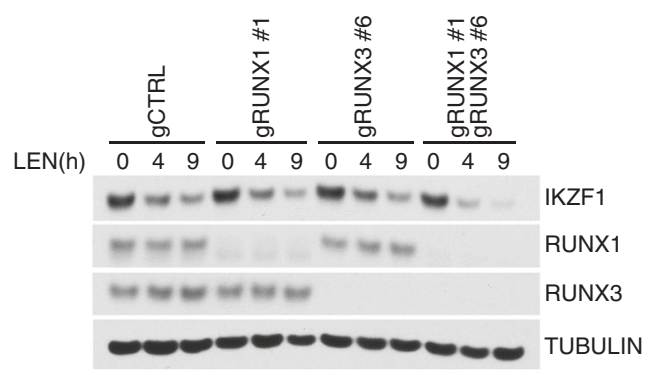

b
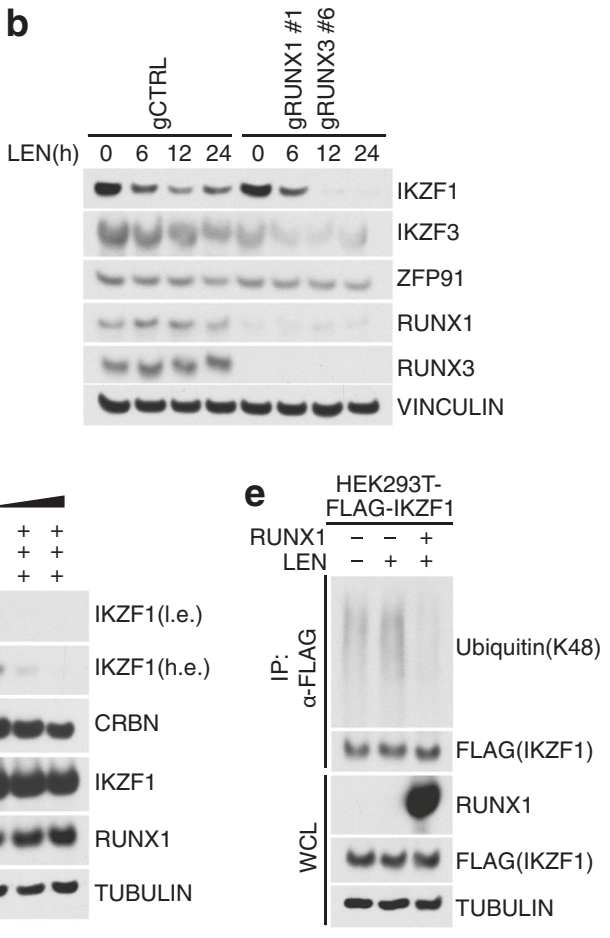
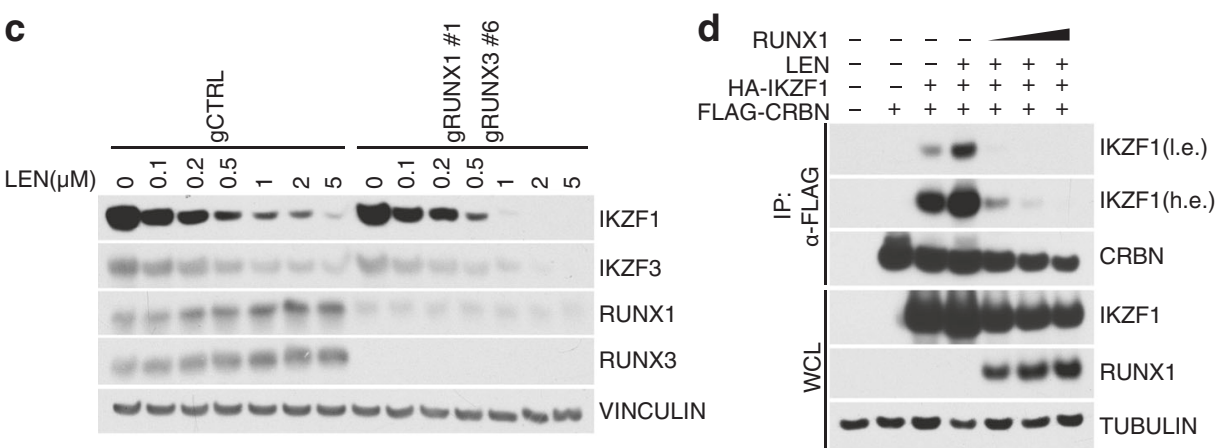

$\mathbf{f}$

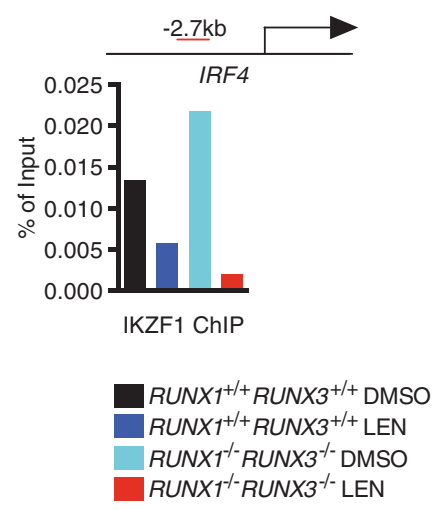

g
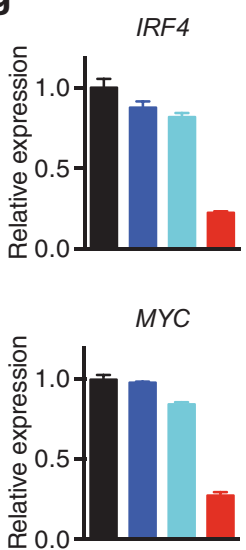

$R U N X 1^{+/+} R U N X 3^{+/+}$DMSO

$R U N X 1^{+/+} R U N X 3^{+/+}$LEN

$R U N X 1^{-/-} R U N X 3^{-/-}$DMSO

$R U N X 1^{-1-} R U N X 3^{-/-}$LEN h $\rightarrow$ RUNX+1/+ RUNX3 $3^{+/+}$

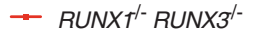

DMSO

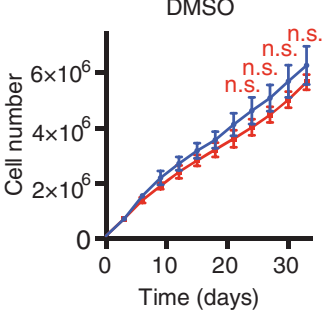

$0.1 \mu \mathrm{M}$ LEN

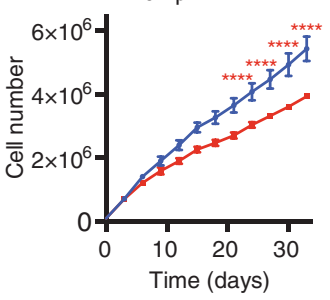

Fig. 3 RUNXs inhibit CRBN-dependent IKZF1 and IKZF3 degradation. a Immunoblot analysis of whole cell lysates from OPM-1 cells treated with $1 \mu \mathrm{M}$ lenalidomide for the indicated durations of time. $\mathbf{b}$ Same as in (a) except that OPM-1 cells were treated with lenalidomide for the indicated time points. c Immunoblot analysis of whole cell lysates from OPM-1 cells treated with at the indicated concentrations of lenalidomide for $36 \mathrm{~h}$. d HEK293T cells were transfected with FLAG-CRBN, HA-IKZF1, and un-tagged RUNX1. Where indicated, cells were treated with $2 \mu \mathrm{M}$ lenalidomide for $6 \mathrm{~h}$ before harvesting. The cell extracts were subjected to anti-FLAG IP and the immunocomplexes were probed with antibodies to the indicated proteins. A low exposure (1.e.) and high exposure (h.e.) are shown for IKZF1. e Same as in d except that HEK293T cells were transfected with
FLAG-IKZF1 and un-tagged RUNX1. f Chromatin immunoprecipitations of IKZF1 in OPM-1 cells coupled with qRT-PCR using primers for IRF4 promoter under the indicated conditions. Red bar shows the distance between the primers and transcription start site of IRF4 $(n=2$ independent experiments). $\mathrm{g}$ Levels of IRF4 and MYC mRNA were analyzed by qRT-PCR under the indicated conditions (mean \pm s.d., $n=2$ independent measurements). h Cell counts of GFP/Cherry-sorted OPM-1 RUNX1 $1^{+/+} R U N X 3^{+/+}$or $R U N X 1^{-/-} R U N X 3^{-/-}$cells grown in media containing DMSO or $0.1 \mu \mathrm{M}$ lenalidomide (mean \pm s.d., $n=3$ independent experiments, two-way ANOVA, n.s., not significant, $* * * * P \leq 0.0001)$. Unless otherwise noted, immunoblots are representative of three independent experiments 

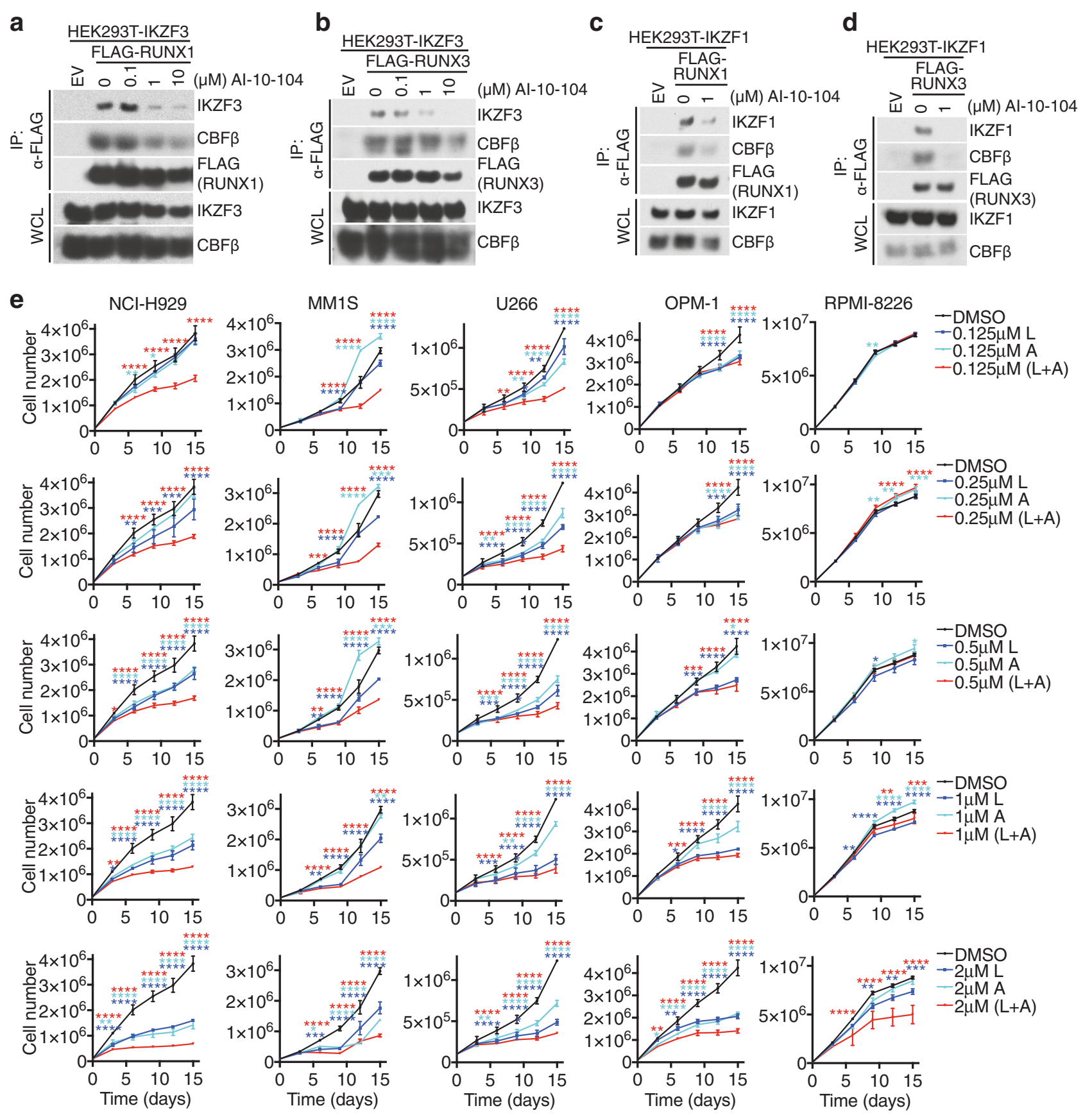

$\mathbf{f}$
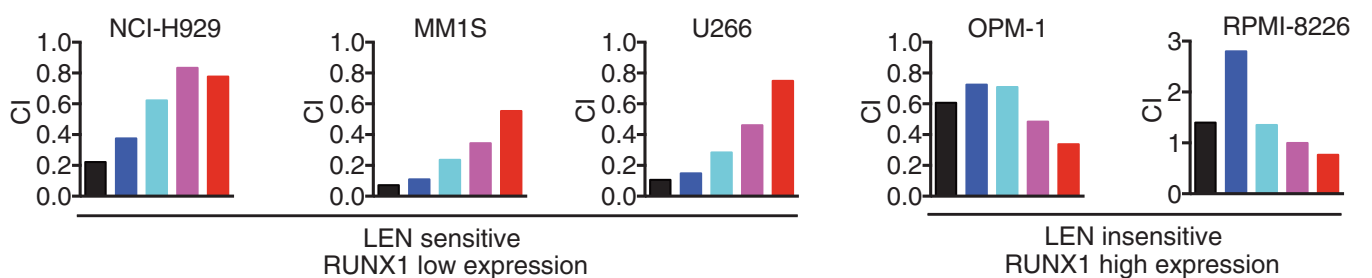

$0.125 \mu \mathrm{M}(\mathrm{L}+\mathrm{A})$

$0.25 \mu \mathrm{M}(\mathrm{L}+\mathrm{A})$

$0.5 \mu \mathrm{M}(\mathrm{L}+\mathrm{A})$

$1 \mu \mathrm{M}(\mathrm{L}+\mathrm{A})$

$2 \mu \mathrm{M}(\mathrm{L}+\mathrm{A})$ 
Fig. 4 Small molecule AI-10-104 can dissociate the IKZFs-RUNXs complex and potentiate the anti-proliferative effect of lenalidomide. a HEK293T cells stably expressing IKZF3 were transfected with constructs encoding an empty vector or FLAG-tagged RUNX1 and treated with increasing amounts of RUNX inhibitor (AI-10-104). Immunoprecipitated FLAG-tagged RUNX1 was probed with antibodies to the indicated proteins. b Same as in (a), except that FLAG-tagged RUNX3 was used. c HEK293T cells stably expressing IKZF1 were transfected with constructs encoding an empty vector or FLAG-tagged RUNX1 and treated with DMSO or $1 \mu \mathrm{M}$ RUNX inhibitor (AI-10-104). Immunoprecipitated FLAG-tagged RUNX1 was probed with antibodies to the indicated proteins. d Same as in (c), except that FLAGtagged RUNX3 was used. e Cell counts of the indicated multiple myeloma cell lines grown in media containing the indicated concentration of lenalidomide (L), RUNX inhibitor AI-10-104 (A), and combination (mean \pm s.d., $n=3$ independent experiments, two-way ANOVA, $* P$-value $\leq 0.05 ; \quad * * P$-value $\leq 0.01 ; * * * P$ value $\leq 0.001$; $* * * * P$-value $\leq 0.0001)$. f Combination indexes (CI) of lenalidomide (L) and RUNX inhibitor AI-10-104 (A) in the indicated multiple myeloma cell lines are shown. Unless otherwise noted, immunoblots are representative of three independent experiments

enhanced IKZFs degradation upon lenalidomide treatment (Supplementary Fig. 4d, e).

Altogether, our data show that chemical inhibition of RUNX proteins increases the cytotoxic effect of lenalidomide in MM cell lines.

\section{RUNX inhibition potentiates the transcriptional response induced by lenalidomide}

To understand the molecular basis for the efficacy of the combination regiment of lenalidomide and RUNX inhibition in treating myeloma, we profiled gene expression changes by RNA sequencing of OPM-1 cells (Supplementary Tables 2, 3 and 4). To this end, we treated cells with $0.1 \mu \mathrm{M}$ lenalidomide or $1 \mu \mathrm{M}$ AI-10-104 and both in combination. As shown previously, single treatments at the indicated concentrations are not effective in blocking OPM1 proliferation (Fig. 4e). Notably, low doses of lenalidomide resulted in deregulation of 69 genes as compared to DMSO counterparts while RUNX inhibition resulted in changes in 38 genes. Importantly, the combination of drugs promoted deregulation of 105 genes (Fig. 5a, b and Supplementary Fig. 5) with upregulation of 60 genes specifically (Fig. 5b). Gene ontology (GO) analysis revealed that the most consistent signatures upregulated by combining lenalidomide and RUNX inhibitor were those associated with interferon signaling, immune response, and inflammatory response (Fig. 5c). Similarly, gene set enrichment analysis (GSEA) revealed significant expression of interferon response gene signatures (Fig. 5d). Specifically, OPM-1 cells treated with the combination of drugs upregulated genes related to interferon (IFN) response relative to either agent alone, including HLA-DQA1, HLA-DRB1, CD74, OAS2, ISG15, NMI, IFIHI, and EPSTII (Fig. 5e).
These observations, coupled with the fact that myeloma cells are more sensitive to the combination of lenalidomide and RUNX inhibitor treatment prompted us to conclude that inhibition of RUNXs enhances the cellular response to lenalidomide, such that lower doses of lenalidomide are able to achieve a therapeutic effect.

\section{Chemical inhibition of RUNXs potentiates lenalidomide toxicity in primary MM cells}

We evaluated primary myeloma samples for their sensitivity to combinatorial therapy consisting of RUNX inhibitor and lenalidomide. Primary myeloma cells were isolated from the iliac crest of patients and subjected to CD138-positive purification. Treatment of diagnostic and relapsed myeloma samples (Fig. 6b) with AI-10-104 or lenalidomide alone resulted in minimal or no change in cell viability when compared to DMSO (Fig. 6a). The combinatorial treatment of AI-10-104 and lenalidomide instead displayed a significant inhibitory effect on the viability of primary myeloma samples. These data are in line with our previous observations in $\mathrm{MM}$ cell lines, where single treatment at low doses of lenalidomide or AI-10-104 did not reduce cell viability as compared with DMSO (Fig. 4e). Again, combination of the two drugs effectively reduced cell viability. Of note, the cytotoxic effect of the lenalidomide and AI-10104 combination regiment was not dependent on patient treatment history (Fig. 6b).

Importantly, treatment of normal human hematopoietic cells with AI-10-104 resulted in an average $\mathrm{IC}_{50}$ of $\sim 15 \mu \mathrm{M}$ $[36,37]$, which greatly exceeded the toxic concentration for MM cell lines and MM primary cells. Thus, RUNX inhibition in myeloma patients should not result in toxic adverse effects on normal hematopoietic stem and progenitor cells. Our data suggest that a therapeutic window may exist for the combinatorial therapy of AI-10-104 derivatives and lenalidomide in MM patients.

\section{Discussion}

Here, we report for the first time that the transcription factors IKZF1 and IKZF3 are physically associated with the RUNX1 and RUNX3 proteins, master regulators of hematopoiesis [38]. IKZFs utilize the N-terminal DNA-binding ZnFs to interact with the AD and ID in RUNX1 and form a trimeric complex with RUNX1-binding partner CBF $\beta$. Our findings are particularly interesting when compared with the anti-myeloma effect of the IMiD drug, lenalidomide. Lenalidomide binds CRBN, a CUL4 E3 ubiquitin ligase, and promotes the ubiquitylation and proteolysis of IKZF1 and IKZF3 $[8,9]$. We show that interaction with RUNX1 results in the inhibition of IKZF1 ubiquitylation and 

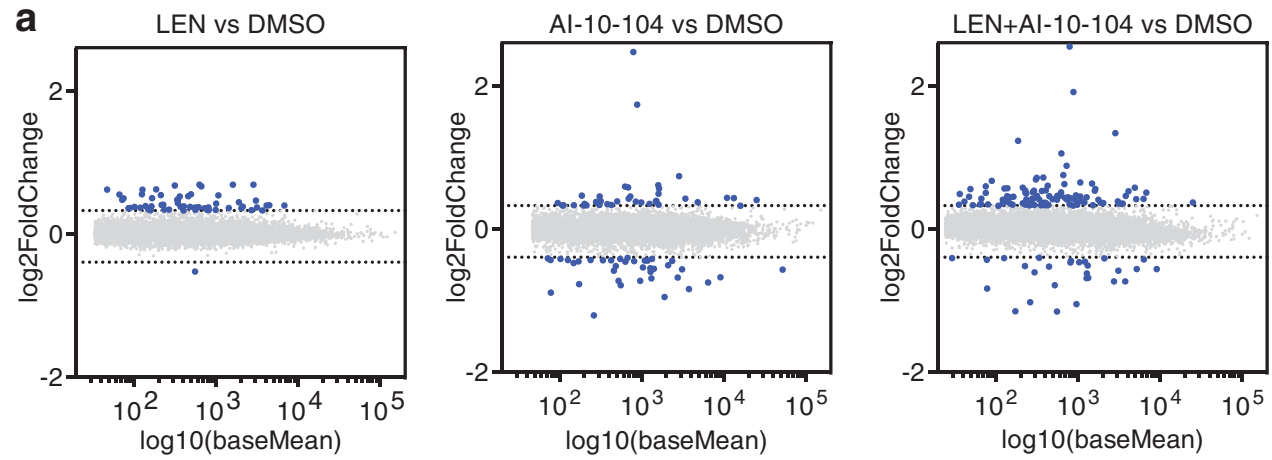

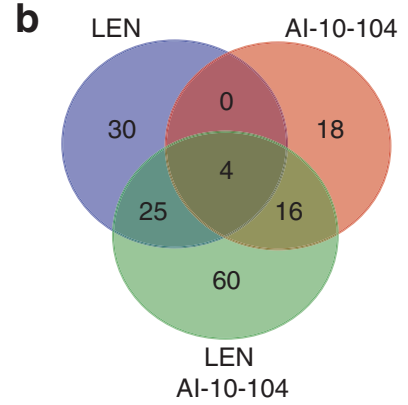

d

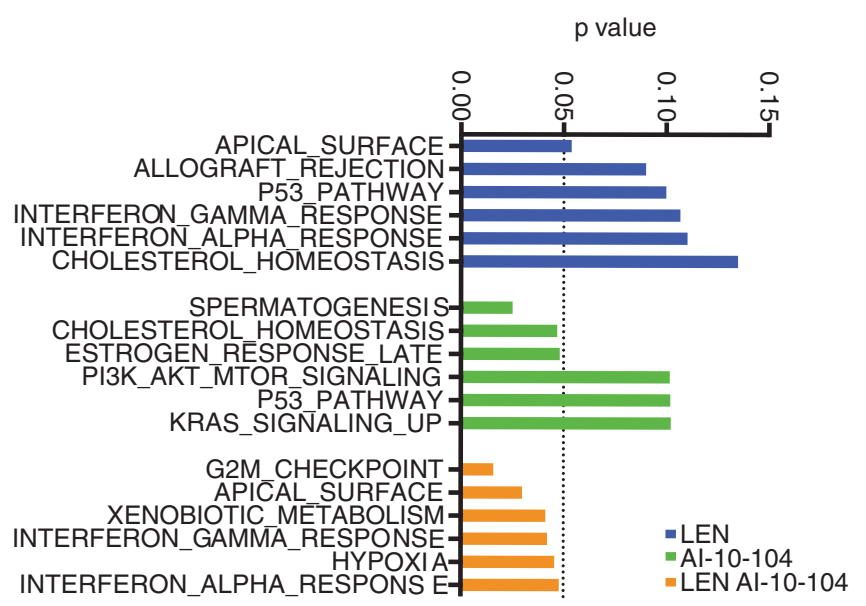

regulation of immune response defense response to virus pos. regul. of transcrip. from RNA pol II promoter
negative regulation of cell proliferation inflammatory response
inf cell proliferation regulation of $\mathrm{TGFb}$ receptor signaling pathway

type I interferon signaling pathway antigen processing and presentation immune response
INFg signaling pathway

$$
\begin{aligned}
& 1,0,0,0,0,0,0,0,0,0,0 \\
& \log 10(\mathrm{p} \text { value) }
\end{aligned}
$$
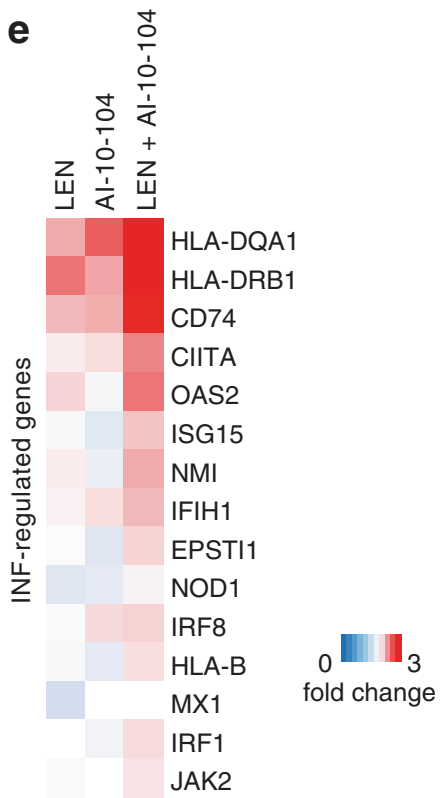

Fig. 5 RUNX inhibition potentiates the transcriptional response induced by lenalidomide. a Fold-change plot of gene expression levels in OPM- 1 cells treated with $0.1 \mu \mathrm{M}$ lenalidomide (LEN), $1 \mu \mathrm{M}$ RUNX inhibitor (AI-10-104), or combination for $48 \mathrm{~h}$ and compared to DMSO ( $n=3$ independent experiments, DeSeq2). b Venn diagram showing the overlap of genes up-regulated or down-regulated by lenalidomide, AI-10-104, and combination and compared to DMSO ( $n=3$ independent experiments, $P \leq 0.05$ ). c Gene ontology (GO) analysis of genes regulated by the combination of lenalidomide and

degradation via the CRBN/lenalidomide complex. Mapping analysis reveals that RUNX1 interacts with the N-terminal ZnFs of IKZF1, a domain also involved in binding to CRBN. Therefore, it is reasonable to conclude that RUNX1
AI-10-104. Bar plot for the $-\log _{10}$ of the $P$ value of the top 10 enriched GO terms of genes regulated is shown. d $P$-value graph of GSEA-enrichment signatures of differentially expressed genes in OPM-1 cells treated with $0.1 \mu \mathrm{M}$ lenalidomide (LEN), $1 \mu \mathrm{M}$ RUNX inhibitor (AI-10-104), or combination and compared to DMSO. e Heat map showing the relative expression of selected interferon signaling genes in OPM- 1 cells treated with $0.1 \mu \mathrm{M}$ lenalidomide (LEN), $1 \mu \mathrm{M}$ RUNX inhibitor (AI-10-104), or combination and compared to DMSO (mean, $n=3$ independent experiments, $P \leq 0.05$ )

and CRBN compete for IKZF1 interaction and the ablation of RUNX1 and RUNX3 results in enhanced degradation of IKZF1 and IKZF3 upon exposure to lenalidomide. Consequently, $R U N X 1^{-/-} R U N X 3^{-/-} \mathrm{MM}$ cells display toxicity to 
Fig. 6 Chemical inhibition of RUNXs potentiates lenalidomide toxicity in primary multiple myeloma cells. a

Primary MM cells were treated with DMSO, $0.1 \mu \mathrm{M}$

lenalidomide, $1 \mu \mathrm{M}$ AI-10-104, and combination. Every 2 days, viable cells were counted using Trypan blue exclusion assay. b The historical treatments of five MM patients are shown
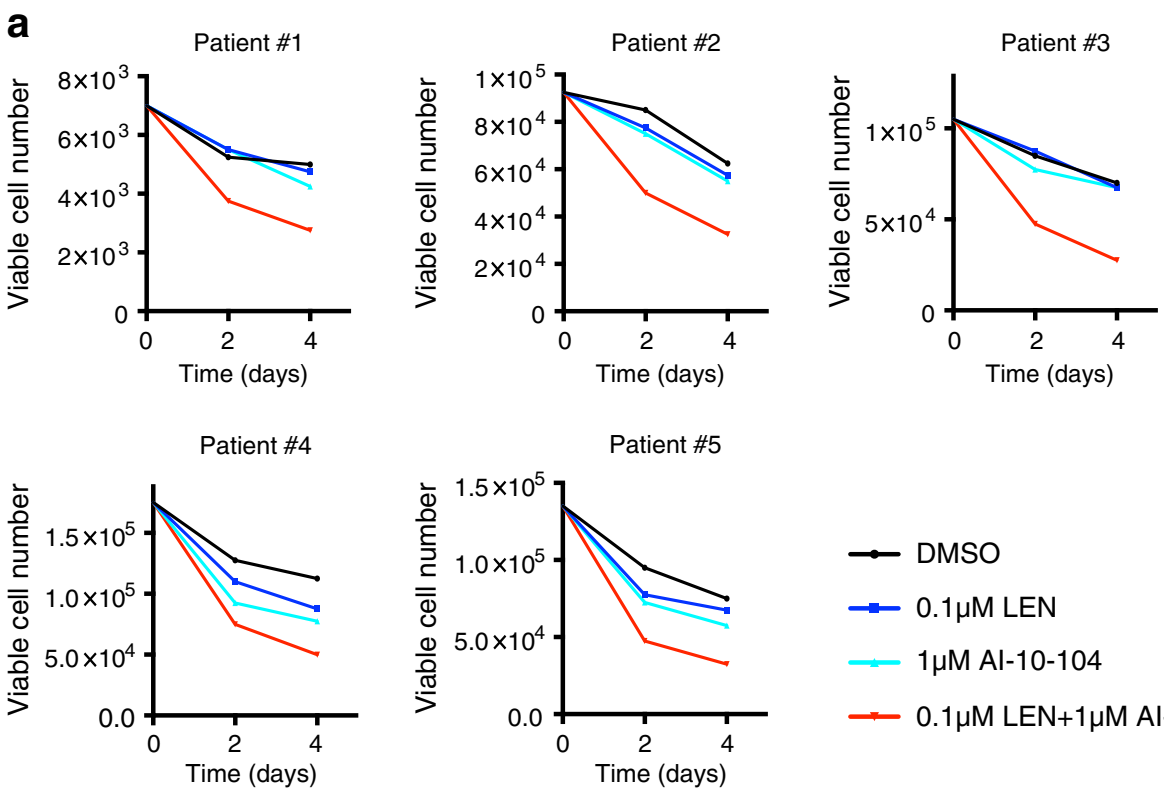

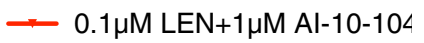

b

\begin{tabular}{cc}
\hline $\begin{array}{c}\text { Patient } \\
\text { No. }\end{array}$ & Historical treatment \\
\hline 1 & $\begin{array}{c}\text { Lenalidomide, Pomalidomide, } \\
\text { Bortezomib, Carfilzomib, } \\
\text { Dexamethasone, Daratumumab, } \\
\text { Cisplatin, Doxorubicin, } \\
\text { Cyclophosphamide, Etoposide }\end{array}$ \\
\hline 2 & None \\
\hline 3 & $\begin{array}{c}\text { Lenalidomide, Pomalidomide, } \\
\text { Dexamethasone, Daratumumab, } \\
\text { Doxorubicin, Cyclophosphamide, } \\
\text { CAR-T BCMA }\end{array}$ \\
\hline 4 & None \\
\hline 5 & None \\
\hline
\end{tabular}

nanomolar concentrations of lenalidomide. Importantly, the enhanced degradation of the IKZF proteins in the $R U N X 1^{-1-}$ $R U N X 3^{-/-}$cells occurs within 4-12 h; time points at which no difference of cell viability is noticeable.

The cellular signaling programs responsible for CRBNIMiD-mediated myeloma cytotoxicity are associated with upregulation of the interferon signaling pathway $[8,9,12$, 32]. In line with the cytotoxic effect in MM cells, RUNX inhibitor potentiates the transcriptional response induced by lenalidomide culminating in the activation of interferon response genes. Thus, deregulation of RUNXs axis could represent an avenue to enhance the anti-myeloma effects of the IMiDs. Further investigation would be necessary to uncover non-cell autonomous mechanisms of this combination therapy; for instance, how lenalidomide and AI-10-104 affect the interaction between myeloma and stroma cells in the bone marrow [39].

Introduction of proteasome inhibitors (bortezomib and carfilzomib) and IMiDs have changed the treatment paradigm for myeloma [40-42]. However, myeloma remains incurable and new treatments are currently being studied. Here, we show that inhibition of RUNXs via the small molecule inhibitor AI-10-104 [36] potentiates the cytotoxic effect of lenalidomide in MM cells. RUNX1 downregulation was previously suggested as a possible therapeutic avenue for the treatment of MM [43]. Furthermore, previous evidence points out a role for RUNX2 in promoting expression of multiple metastatic genes and favoring homing of MM cells to the bone [44]. Our data suggest that RUNX proteins are dispensable with regard to the 
proliferation potential of $\mathrm{MM}$ cells, and the drugs that achieve pan-inhibition of the RUNX proteins might act as an intervention point for the treatment of MM, particularly in combination with low doses of lenalidomide (Supplementary Fig. 6).

\section{Methods}

\section{Cell culture}

ARP-1, OPM-1, KMS-11, RPMI-8226, NCI-H929, and U266 were maintained in RPMI1640 media containing 10\% fetal bovine serum (FBS). HEK293T cells were maintained in Dulbecco's modified Eagle's media (DMEM) containing $10 \%$ FBS. Primary myeloma cells were obtained from the iliac crest aspirates from MM patients. All patients provided written informed consent under a research sample collection protocol approved by the University of Pennsylvania Institutional Review Board. CD $138^{+}$cells were isolated with Ficoll Paque Plus (GE Healthcare, \#17-1440-02) and $\mathrm{CD} 138^{+}$Microbeads (Miltonic Biotic, \#130-051-301) according to the manufacturer's protocol. Primary CD138 cells were maintained in RPMI1640 media containing 50\% own serum from the corresponding patients.

\section{Reagents}

The following antibodies were used: anti-FLAG (Sigma, F7425), anti-IKZF3 (Cell Signaling, \#15103), anti-IKZF1 (Cell Signaling, \#9034), anti-RUNX1 (Abcam, ab92336), anti-RUNX3 (Cell Signaling, \#9647), anti-CBF $\beta$ (Santa Cruz, sc-20693), anti-CRBN (Sigma, HPA045910), anti-ubiquitin (K48) (EMD Millipore, \#05-1307), antiTUBULIN (Santa Cruz, sc-8035), anti-VINCULIN (Santa Cruz, sc-73614), anti-Rabbit IgG-HRP (GE Healthcare, NA934V), and anti-mouse IgG-HRP (GE Healthcare, NA931V). The following agarose beads were used: antiFLAG M2 Affinity Gel (Sigma, A2220) and Glutathione Sepharose 4B (GE Healthcare, \#17075601). The following in vitro translation kit was used: TNT T7 Coupled Reticulocyte Lysate Systems (Promega, L4610). Benzonase was used according to manufacturer (Sigma, E1014). The following compounds were used: Lenalidomide (Sigma, CDS022536), Pomalidomide (Sigma, P0018), Bortezomib (Millennium Pharmaceuticals), and AI-10-104 was kindly provided by Dr. John H. Bushweller.

\section{Gene silencing by siRNA}

siRNA sequences are as follows: siRUNX1, 5'-CCUCGAA GACAUCGGCAGAAA-3'; siRUNX2, 5'-CUCUGCACC AAGUCCUUUU-3'; and siRUNX3, 5'-CCUUCAAGGU
GGUGGCAUU- ${ }^{\prime}$. The following siRNAs were obtained from Dharmacon: siIKZF1 \#1 (D-019092-01), siIKZF1 \#2 (D-019092-02), siIKZF1 \#3, (D-019092-03), siIKZF1 \#4 (D-019092-04), siIKZF3 \#3 (D-006945-03), siIKZF3 \#4 (D-006945-04), siIKZF3 \#17 (D-006945-17), siIKZF3 \#18 (D-006945-18), and ON-TARGETplus. Non-targeting control siRNAs were obtained from Dharmacon (D-001810-01-20).

\section{Plasmids}

The detailed information regarding plasmid constructions is available by request. And the target sequences used to knock-out human RUNXs are as follows: hRUNX1_ gRNA\#1 Fwd: ATGAGCGAGGCGTTGCCGCT, Rev: AGCGGCAACGCCTCGCTCAT; hRUNX3_gRNA\#6 Fwd: GCCCGAGGTGCGCTCGATGG, Rev: CCATCGA GCGCACCTCGGGC; and control_gRNA Fwd: CTTCG AAATGTCCGTTCGGT, Rev: ACCGAACGGACATTT CGAAG.

\section{Immunoprecipitation and immunoblot}

Cells were lysed in NP-40 buffer (0.1\% NP-40, $15 \mathrm{mM}$ Tris-HCl pH7.4, $1 \mathrm{mM}$ EDTA, $150 \mathrm{mM} \mathrm{NaCl}, 1 \mathrm{mM}$ $\mathrm{MgCl}_{2}, 10 \%$ Glycerol) containing protease inhibitors (Sigma, \#11697498001) and the lysates were incubated with anti-FLAG Gel at $4{ }^{\circ} \mathrm{C}$ overnight. After washing, the anti-FLAG Gel was mixed with Laemmli buffer and boiled at $95{ }^{\circ} \mathrm{C}$ for $5 \mathrm{~min}$. After SDS-PAGE electrophoresis and transfer, primary antibodies and HRP-linked secondary antibodies were incubated with the membrane for $1 \mathrm{~h}$ at room temperature and overnight at $4{ }^{\circ} \mathrm{C}$, respectively. Where indicated, anti-FLAG immunoprecipitates were resuspended in $50 \mu \mathrm{l}$ of NP-40 buffer, incubated with $1 \mu \mathrm{l}$ of Benzonase for $30 \mathrm{~min}$ on ice, and further washed twice. For GST pull-down, GST-tagged proteins were incubated with in vitro translated FLAG-tagged proteins at $4{ }^{\circ} \mathrm{C}$ overnight. After washing with PBS-T three times and PBS once, the membrane was detected by the chemiluminescence system (Thermo Fisher Scientific, \#32106).

\section{Chromatin immunoprecipitation}

Cells were cross-linked using $1 \%$ formaldehyde for $5 \mathrm{~min}$. Then cells were incubated with $125 \mathrm{mM}$ glycine for $5 \mathrm{~min}$. After centrifugation at $1000 \mathrm{rpm}, 4{ }^{\circ} \mathrm{C}$ for $5 \mathrm{~min}$, cell pellets were washed with PBS once and lysed with cell lysis buffer (0.2\% NP-40, $100 \mathrm{mM}$ Tris- $\mathrm{HCl} \mathrm{pH} 8,10 \mathrm{mM} \mathrm{NaCl})$ for $10 \mathrm{~min}$ on ice. After centrifugation at $2500 \mathrm{rpm}, 4^{\circ} \mathrm{C}$ for $5 \mathrm{~min}$, the pellets were re-suspended with nucleus lysis buffer (0.1\% SDS, $50 \mathrm{mM}$ Tris-HCl pH8, $10 \mathrm{mM}$ EDTA) to do sonication using the Covaris S220 system (Thermo 
Fisher Scientific, \#4465653) according to the manufacturer's protocol. After sonication, the cell lysates were centrifuged at $13,000 \mathrm{rpm}, 4{ }^{\circ} \mathrm{C}$ for $5 \mathrm{~min}$ and the supernatant was collected. Then $10 \mu \mathrm{l}$ of Dynabeads Protein A (Life Technology, \#10001D) were blocked with $1 \mathrm{mg} / \mathrm{ml}$ of BSA at $4{ }^{\circ} \mathrm{C}$ for $1 \mathrm{~h}$ and $30 \mu \mathrm{l}$ of Dynabeads were washed with PBS for three times and incubated with $5 \mu \mathrm{g}$ of antibodies at $4{ }^{\circ} \mathrm{C}$ for $4 \mathrm{~h}$. Three hundred microliters of sonicated nucleus lysates were incubated with $10 \mu \mathrm{l}$ of BSA-blocked Dynabeads at $4{ }^{\circ} \mathrm{C}$ for $3 \mathrm{~h}$. After $3 \mathrm{~h}$, the precleared $300 \mu \mathrm{l}$ of nucleus lysates and $700 \mu \mathrm{l}$ of IP buffer (0.01\% SDS, $1 \%$ TritonX-100, $20 \mathrm{mM}$ Tris- $\mathrm{HCl} \mathrm{pH} 8$, $2 \mathrm{mM}$ EDTA, $150 \mathrm{mM} \mathrm{NaCl}$ ) was incubated with $30 \mu \mathrm{l}$ of antibody-conjugated Dynabeads at $4{ }^{\circ} \mathrm{C}$ overnight. Then the Dynabeads were washed twice with low salt IP wash buffer (0.01\% SDS, $1 \%$ TritonX-100, $20 \mathrm{mM}$ Tris-HCl pH8, $2 \mathrm{mM}$ EDTA, $50 \mathrm{mM} \mathrm{NaCl}$ ), twice with medium salt IP wash buffer $(0.01 \%$ SDS, $1 \%$ TritonX-100, $20 \mathrm{mM}$ Tris$\mathrm{HCl} \mathrm{pH} 8,2 \mathrm{mM}$ EDTA, $300 \mathrm{mM} \mathrm{NaCl}$ ), and twice with high salt IP wash buffer $(0.01 \%$ SDS, $1 \%$ TritonX-100, $20 \mathrm{mM}$ Tris- $\mathrm{HCl} \mathrm{pH} 8,2 \mathrm{mM}$ EDTA, $500 \mathrm{mM} \mathrm{NaCl}$ ). The Dynabeads were washed once with $\mathrm{LiCl}$ buffer (1\% NP-40, $1 \%$ deoxycholic acid, $10 \mathrm{mM}$ Tris- $\mathrm{HCl} \mathrm{pH} 8,1 \mathrm{mM}$ EDTA, $0.25 \mathrm{M} \mathrm{LiCl})$ and once with TE buffer $(10 \mathrm{mM}$ Tris-HCl pH8, $1 \mathrm{mM}$ EDTA). The chromatin reverse cross-linking and DNA elution was then conducted using IPure kit (diagenode, C03010015) according to the manufacturer's protocol.

\section{Quantitative real-time PCR (qRT-qPCR)}

qRT-PCR was conducted with SYBR Green PCR Master Mix (Thermo Fisher Scientific, \#4309155) and the comparative $\mathrm{C}_{\mathrm{T}}$ method was utilized for relative quantification on the ViiA 7 real-time PCR system (Thermo Fisher Scientific). The primer sequences are as follows: for ChIP analysis, hIRF4 Fwd: 5'-AGTTGCAGGTTGACCTACGG3', Rev: 5'-AGCTTTCACCCGTTGAGCTT-3'; for mRNA analysis, hIRF4 Fwd: 5'-ATTCCAGGTGACTCTATG-3', Rev: 5'-GATTGCTGATGTGTTCTG-3', hMYC Fwd: 5'CCACCAGCAGCGACTCTG-3', Rev: 5'-AGCCTGCCT CTTTTCCAC-3', hGAPDH Fwd: 5'-GGAGCGAGATCC CTCCAAAAT-3', Rev: 5'-GGCTGTTGTCATACTTCTC ATGG-3'.

\section{MudPIT analysis}

TCA-precipitates were urea-denatured, reduced, alkylated, and digested with endoproteinase Lys-C (Roche), followed by modified trypsin (Roche) [45, 46]. Peptide mixtures were loaded onto $100-\mu \mathrm{m}$-fused silica microcapillary columns packed with 5- $\mu \mathrm{m} \mathrm{C18}$ reverse phase (Aqua, Phenomenex), strong cation exchange particles (Luna, Phenomenex) [47].
Loaded microcapillary columns were placed in-line with a Quaternary Agilent 1100 series HPLC pump and a LTQ linear ion trap mass spectrometer equipped with a nano-LC electrospray ionization source (Thermo Scientific). Fullyautomated 10-step MudPIT runs were carried out on the electrosprayed peptides, as described [48]. Tandem mass (MS/MS) spectra were interpreted using SEQUEST [49] against a database of 72,956 sequences, consisting of 72,968 non-redundant human proteins (downloaded from NCBI on 2015-03-25), 148 usual contaminants (such as human keratins, IgGs, and proteolytic enzymes), and to estimate false-discovery rates, 73,091 randomized aminoacid sequences derived from each non-redundant protein entry. Peptide/spectrum matches were sorted and selected using DTASelect with the following criteria set: spectra/ peptide matches were only retained if they had a DeltCN of at least 0.08 and a minimum xcorr of 1.8 for singly charged, 2.5 for doubly charged, and 3.5 for triply charged spectra. In addition, peptides had to be fully tryptic and at least seven amino acids long. Combining all runs, proteins had to be detected by at least two such peptides, or one peptide with two independent spectra. Under these criteria the averagel FDRs at the protein and spectral levels were $0.5 \pm 0.3 \%$ and $0.06 \pm 0.05 \%$, respectively. Peptide hits from multiple runs were compared using CONTRAST [50]. To estimate relative protein levels, normalized spectral abundance factors (NSAFs) were calculated for each detected protein, as described [51-53]. Original data underlying this manuscript can be accessed from the Stowers Original Data Repository at http://www.stowers.org/research/publications/libpb-1237.

\section{Transfection and retrovirus-mediated gene transfer}

HEK293T cells were transfected with plasmids using polyethylenimine (PEI) (Polysciences, \#24765). For retrovirus and lentivirus production, GP2-293 packaging cells (Clontech) or pCMV-DeltaR8.2 were used. After $72 \mathrm{~h}$ of transfection, the virus-containing media was collected and used to spin-infect the cells at $1800 \mathrm{rpm}$ for $40 \mathrm{~min}$. Cells were then incubated with the viral supernatant overnight. For siRNA transfection, the Neon transfection system (Thermo Fisher Scientific, \#MPK5000) was used.

\section{RNA-Seq}

Total RNA was extracted from OPM-1 cells using RNeasy Mini Kit (QIAGEN, \#74104) and polyA+ transcripts were isolated with NEBNext Poly(A) mRNA Magnetic Isolation Module (NEB, \#7490). RNA-Seq libraries were prepared with NEBNext Ultra Directional RNA Library Prep Kit for Illumina (NEB, E7420). Three biological replicates were sequenced on a NextSeq 500 (Illumina) at a depth of at least $2 \times 10^{7}$ reads each. Reads were mapped and analyzed with a 
bioinformatic pipeline based on STAR, SAMTOOLS, and the $\mathrm{R}$ packages DEGseq and DEseq2. We used human genome version GRCh38. GO analyses were performed using version 6.8 of the DAVID web server. GSEA analyses were performed using pre-ranked GSEA using a weighted scoring [54]. Original data can be accessed at GSE123660.

\section{Cell proliferation assay}

OPM-1, RPMI-8226, NCI-H929, MM1S, and U266 cells were cultured in six-well plates. The initial cell number was 100,000 cells per well and the cell numbers were counted and re-plated at the initial concentration every 3 days. Lenalidomide and AI-10-104 were added into the culture media after dilution every 3 days. The cell counting was conducted with Z2 Coulter Counter Analyzer (Beckman Coulter, \#6605700).

\section{Trypan blue exclusion assay}

Fifteen microliters of 0.4\% Trypan blue solution (Cellgro, \#25-900-CI) was mixed with $15 \mu \mathrm{l}$ of $\mathrm{CD} 138^{+}$cell suspension and incubated for $2 \mathrm{~min}$ at room temperature. The viable cell numbers were counted using a hemocytometer (Fisher Scientific, \#02-671-10).

\section{Statistical analyses}

All statistical analyses were performed with Prism 6 (GraphPad), and sample sizes and reproducibility for each figure are shown in the figure legends. Student's $t$-tests and one-way or two-way ANOVA analyses were performed and indicated in the figure legends. CIs were calculated with CompuSyn software. All graphs show mean values with error bars signifying s.d. as indicated in the figure legends.

\section{Data availability}

The authors declare that the data supporting the findings of this study are available upon reasonable requests.

\begin{abstract}
Acknowledgements The authors thank Ashley N. Hughes and Grant Grothusen for critically reading the manuscript and Nancy A. Speck for kindly providing RUNX1 constructs. This work was supported in part by grants R00-CA166181-04 and R01-CA207513-01 from the National Cancer Institute to L.B.
\end{abstract}

Author contributions LB conceived, directed the project, and oversaw the results. NZ designed and performed most experiments. XYZ helped NZ with experiments in Figs. 3i, 4c, S2a, S3. AGU performed experiments in Figs. 1a, 1b, 2b, 2c. AS, LF and MPW performed the mass spectrometry analysis of the IKZF complexes purified by AG-U, $\mathrm{DV}, \mathrm{ALG}, \mathrm{LBe}$ isolated the primary myeloma sample. RC performed experiments in Fig. 4e. AI and JHB provided the AI-10-104. LB and NZ wrote the manuscript.

\section{Compliance with ethical standards}

Conflict of interest The authors declare that they have no conflict of interest.

Publisher's note: Springer Nature remains neutral with regard to jurisdictional claims in published maps and institutional affiliations.

Open Access This article is licensed under a Creative Commons Attribution 4.0 International License, which permits use, sharing, adaptation, distribution and reproduction in any medium or format, as long as you give appropriate credit to the original author(s) and the source, provide a link to the Creative Commons license, and indicate if changes were made. The images or other third party material in this article are included in the article's Creative Commons license, unless indicated otherwise in a credit line to the material. If material is not included in the article's Creative Commons license and your intended use is not permitted by statutory regulation or exceeds the permitted use, you will need to obtain permission directly from the copyright holder. To view a copy of this license, visit http://creativecommons. org/licenses/by/4.0/.

\section{References}

1. McCarthy PL, Owzar K, Hofmeister CC, Hurd DD, Hassoun H, Richardson PG, et al. Lenalidomide after stem-cell transplantation for multiple myeloma. N Engl J Med. 2012;366:1770-81.

2. Palumbo A, Anderson K. MEDICAL PROGRESS Multiple Myeloma. New England Journal of Medicine. 2011;364:1046-60.

3. John LB, Ward AC. The Ikaros gene family: transcriptional regulators of hematopoiesis and immunity. Mol Immunol. 2011;48:1272-8.

4. Yoshida T, Georgopoulos K. Ikaros fingers on lymphocyte differentiation. Int J Hematol. 2014;100:220-9.

5. Georgopoulos K, Bigby M, Wang JH, Molnar A, Wu P, Winandy $\mathrm{S}$, et al. The Ikaros Gene Is Required for the Development of All Lymphoid Lineages. Cell. 1994;79:143-56.

6. Winandy S, Wu P, Georgopoulos K. A dominant mutation in the Ikaros gene leads to rapid development of leukemia and lymphoma. Cell. 1995;83:289-99.

7. Mullighan CG, Goorha S, Radtke I, Miller CB, Coustan-Smith E, Dalton JD, et al. Genome-wide analysis of genetic alterations in acute lymphoblastic leukaemia. Nature. 2007;446:758-64.

8. Lu G, Middleton RE, Sun H, Naniong M, Ott CJ, Mitsiades CS, et al. The myeloma drug lenalidomide promotes the cereblon-dependent destruction of Ikaros proteins. Science. 2014;343:305-9.

9. Kronke J, Udeshi ND, Narla A, Grauman P, Hurst SN, McConkey $\mathrm{M}$, et al. Lenalidomide causes selective degradation of IKZF1 and IKZF3 in multiple myeloma cells. Science. 2014;343:301-5.

10. Fischer ES, Bohm K, Lydeard JR, Yang H, Stadler MB, Cavadini $\mathrm{S}$, et al. Structure of the DDB1-CRBN E3 ubiquitin ligase in complex with thalidomide. Nature. 2014;512:49-53.

11. Petzold G, Fischer ES, Thoma NH. Structural basis of lenalidomide-induced CK1alpha degradation by the CRL4(CRBN) ubiquitin ligase. Nature. 2016;532:127-30.

12. Bjorklund CC, Lu L, Kang J, Hagner PR, Havens CG, Amatangelo $\mathrm{M}$, et al. Rate of CRL4(CRBN) substrate Ikaros and Aiolos degradation underlies differential activity of lenalidomide and pomalidomide in multiple myeloma cells by regulation of cMyc and IRF4. Blood Cancer Journal. 2015;5. 
13. Shaffer AL, Emre NC, Lamy L, Ngo VN, Wright G, Xiao W, et al. IRF4 addiction in multiple myeloma. Nature. 2008;454: 226-31.

14. Osato M. Point mutations in the RUNX1/AML1 gene: another actor in RUNX leukemia. Oncogene. 2004;23:4284-96.

15. Song WJ, Sullivan MG, Legare RD, Hutchings S, Tan X, Kufrin D, et al. Haploinsufficiency of CBFA2 causes familial thrombocytopenia with propensity to develop acute myelogenous leukaemia. Nat Genet. 1999;23:166-75.

16. Harada H, Harada Y, Niimi H, Kyo T, Kimura A, Inaba T. High incidence of somatic mutations in the AML1/RUNX1 gene in myelodysplastic syndrome and low blast percentage myeloid leukemia with myelodysplasia. Blood. 2004;103:2316-24.

17. Levanon D, Negreanu V, Bernstein Y, Bar-Am I, Avivi L, Groner Y. AML1, AML2, and AML3, the human members of the runt domain gene-family: cDNA structure, expression, and chromosomal localization. Genomics. 1994;23:425-32.

18. Huang G, Zhang P, Hirai H, Elf S, Yan XM, Chen Z, et al. PU.1 is a major downstream target of AML1 (RUNX1) in adult mouse hematopoiesis (vol 40, pg 51, 2008). Nat Genet. 2008;40:255-255.

19. Okuda T, vanDeursen J, Hiebert SW, Grosveld G, Downing JR. AML1, the target of multiple chromosomal translocations in human leukemia, is essential for normal fetal liver hematopoiesis. Cell. 1996;84:321-30.

20. Zhang J, Jackson AF, Naito T, Dose M, Seavitt J, Liu F, et al. Harnessing of the nucleosome-remodeling-deacetylase complex controls lymphocyte development and prevents leukemogenesis. Nat Immunol. 2011;13:86-94.

21. O'Neill DW, Schoetz SS, Lopez RA, Castle M, Rabinowitz L, Shor $\mathrm{E}$, et al. An ikaros-containing chromatin-remodeling complex in adult-type erythroid cells. Mol Cell Biol. 2000;20:7572-82.

22. Kagoshima H, Shigesada K, Satake M, Ito Y, Miyoshi H, Ohki M, et al. The Runt domain identifies a new family of heteromeric transcriptional regulators. Trends Genet. 1993;9:338-41.

23. Meyers S, Downing JR, Hiebert SW. Identification of AML-1 and the $(8 ; 21)$ translocation protein (AML-1/ETO) as sequence-specific DNA-binding proteins: the runt homology domain is required for DNA binding and protein-protein interactions. Mol Cell Biol. 1993;13:6336-45.

24. McCarty AS, Kleiger G, Eisenberg D, Smale ST. Selective dimerization of a C2H2 zinc finger subfamily. Mol Cell. 2003;11:459-70.

25. Ng SY, Yoshida T, Zhang J, Georgopoulos K. Genome-wide lineage-specific transcriptional networks underscore Ikarosdependent lymphoid priming in hematopoietic stem cells. Immunity. 2009;30:493-507.

26. Molnar A, Georgopoulos K. The Ikaros gene encodes a family of functionally diverse zinc finger DNA-binding proteins. Mol Cell Biol. 1994;14:8292-303.

27. Sun L, Liu A, Georgopoulos K. Zinc finger-mediated protein interactions modulate Ikaros activity, a molecular control of lymphocyte development. EMBO J. 1996;15:5358-69.

28. Petrovick MS, Hiebert SW, Friedman AD, Hetherington CJ, Tenen DG, Zhang DE. Multiple functional domains of AML1: PU.1 and C/EBPalpha synergize with different regions of AML1. Mol Cell Biol. 1998;18:3915-25.

29. Kanno T, Kanno Y, Chen LF, Ogawa E, Kim WY, Ito Y. Intrinsic transcriptional activation-inhibition domains of the polyomavirus enhancer binding protein $2 /$ core binding factor alpha subunit revealed in the presence of the beta subunit. Mol Cell Biol. 1998;18:2444-54.

30. Matheny CJ, Speck ME, Cushing PR, Zhou Y, Corpora T, Regan $\mathrm{M}$, et al. Disease mutations in RUNX1 and RUNX2 create nonfunctional, dominant-negative, or hypomorphic alleles. EMBO J. 2007;26:1163-75.
31. An J, Ponthier CM, Sack R, Seebacher J, Stadler MB, Donovan $\mathrm{KA}$, et al. pSILAC mass spectrometry reveals ZFP91 as IMiDdependent substrate of the CRL4(CRBN) ubiquitin ligase. Nat Commun. 2017;8:15398.

32. Lopez-Girona A, Heintel D, Zhang LH, Mendy D, Gaidarova S, Brady $\mathrm{H}$, et al. Lenalidomide downregulates the cell survival factor, interferon regulatory factor-4, providing a potential mechanistic link for predicting response. Br J Haematol. 2011;154:325-36.

33. Yang Y, Shaffer AL 3rd, Emre NC, Ceribelli M, Zhang M, Wright $\mathrm{G}$, et al. Exploiting synthetic lethality for the therapy of $\mathrm{ABC}$ diffuse large B cell lymphoma. Cancer Cell. 2012;21:723-37.

34. Zhang LH, Kosek J, Wang M, Heise C, Schafer PH, Chopra R. Lenalidomide efficacy in activated B-cell-like subtype diffuse large B-cell lymphoma is dependent upon IRF4 and cereblon expression. Brit J Haematol. 2013;160:487-502.

35. Zhu YX, Braggio E, Shi CX, Bruins LA, Schmidt JE, Van Wier S, et al. Cereblon expression is required for the antimyeloma activity of lenalidomide and pomalidomide. Blood. 2011;118:4771-9.

36. Illendula A, Gilmour J, Grembecka J, Tirumala VSS, Boulton A, Kuntimaddi A, et al. Small Molecule Inhibitor of CBFbeta-RUNX Binding for RUNX Transcription Factor Driven Cancers. EBioMedicine. 2016;8:117-31.

37. Choi A, Illendula A, Pulikkan JA, Roderick JE, Tesell J, Yu J, et al. RUNX1 is required for oncogenic Myb and Myc enhancer activity in T-cell acute lymphoblastic leukemia. Blood. 2017;130:1722-33.

38. Ito Y, Bae SC, Chuang LSH. The RUNX family: developmental regulators in cancer. Nat Rev Cancer. 2015;15:81-95.

39. Gorgun G, Calabrese E, Soydan E, Hideshima T, Perrone G, Bandi $\mathrm{M}$, et al. Immunomodulatory effects of lenalidomide and pomalidomide on interaction of tumor and bone marrow accessory cells in multiple myeloma. Blood. 2010;116:3227-37.

40. Brenner H, Gondos A, Pulte D. Recent major improvement in long-term survival of younger patients with multiple myeloma. Blood. 2008;111:2521-6.

41. Kumar SK, Mikhael JR, Buadi FK, Dingli D, Dispenzieri A, Fonseca R, et al. Management of newly diagnosed symptomatic multiple myeloma: updated Mayo Stratification of Myeloma and Risk-Adapted Therapy (mSMART) consensus guidelines. Mayo Clin Proc. 2009;84:1095-110.

42. Kumar SK, Rajkumar SV, Dispenzieri A, Lacy MQ, Hayman SR, Buadi FK, et al. Improved survival in multiple myeloma and the impact of novel therapies. Blood. 2008;111:2516-20.

43. Choi SJ, Oba T, Callander NS, Jelinek DF, Roodman GDAML1A. and AML-1B regulation of MIP-1alpha expression in multiple myeloma. Blood. 2003;101:3778-83.

44. Trotter TN, Li M, Pan QY, Peker D, Rowan PD, Li J, et al. Myeloma cell-derived Runx2 promotes myeloma progression in bone. Blood. 2015;125:3598-608.

45. Florens L, Washburn MP. Proteomic analysis by multidimensional protein identification technology. Methods Mol Biol. 2006;328:159-75.

46. Washburn MP, Wolters D, Yates JR 3rd. Large-scale analysis of the yeast proteome by multidimensional protein identification technology. Nat Biotechnol. 2001;19:242-7.

47. McDonald WH, Ohi R, Miyamoto DT, Mitchison TJ, Yates JR. Comparison of three directly coupled HPLC MS/MS strategies for identification of proteins from complex mixtures: single-dimension LC-MS/MS, 2-phase MudPIT, and 3-phase MudPIT. Int J Mass Spectrom. 2002;219:245-51.

48. Wang Z, Hill S, Luther JM, Hachey DL, Schey KL. Proteomic analysis of urine exosomes by multidimensional protein identification technology (MudPIT). Proteomics. 2012;12:329. +

49. Eng JK, McCormack AL, Yates JR. An approach to correlate tandem mass spectral data of peptides with amino acid sequences in a protein database. J Am Soc Mass Spectrom. 1994;5:976-89. 
50. Tabb DL, McDonald WH, Yates JR 3rd. DTASelect and Contrast: tools for assembling and comparing protein identifications from shotgun proteomics. J Proteome Res. 2002; $1: 21-6$.

51. Florens L, Carozza MJ, Swanson SK, Fournier M, Coleman MK, Workman JL, et al. Analyzing chromatin remodeling complexes using shotgun proteomics and normalized spectral abundance factors. Methods. 2006;40:303-11.

52. Paoletti AC, Parmely TJ, Tomomori-Sato C, Sato S, Zhu D, Conaway RC, et al. Quantitative proteomic analysis of distinct mammalian Mediator complexes using normalized spectral abundance factors. Proc Natl Acad Sci USA. 2006;103:18928-33.

53. Zybailov B, Mosley AL, Sardiu ME, Coleman MK, Florens L, Washburn MP. Statistical analysis of membrane proteome expression changes in Saccharomyces cerevisiae. J Proteome Res. 2006;5:2339-47.

54. Subramanian A, Tamayo P, Mootha VK, Mukherjee S, Ebert BL, Gillette MA, et al. Gene set enrichment analysis: A knowledgebased approach for interpreting genome-wide expression profiles. P Natl Acad Sci USA. 2005;102:15545-50. 